\title{
Temperature Dependence of Damping and Frequency Shifts of the Scissors Mode of a trapped Bose-Einstein Condensate
}

\author{
Onofrio Maragò, Gerald Hechenblaikner, Eleanor Hodby, and Christopher Foot \\ Clarendon Laboratory, Department of Physics, University of Oxford, \\ Parks Road, Oxford, OX1 3PU, \\ United Kingdom. \\ (October 29, 2018)
}

We have studied the properties of the scissors mode of a trapped Bose-Einstein condensate of ${ }^{87} \mathrm{Rb}$ atoms at finite temperature. We measured a significant shift in the frequency of the mode below the hydrodynamic limit and a strong dependence of the damping rate as the temperature increased. We compared our damping rate results to recent theoretical calculations for other observed collective modes finding a fair agreement. From the frequency measurements we deduce the moment of inertia of the gas and show that it is quenched below the transition point, because of the superfluid nature of the condensed gas.

PACS numbers: 03.75.Fi, 05.30.Jp, 32.80.Pj, 67.90.+z

The experimental discovery of the scissors mode [1], first predicted in a geometrical model [2], has been one of the most exciting findings in nuclear physics during the last two decades (see [3] for a review). According to the geometrical picture, such a mode arises from a counterrotational oscillation of the deformed proton and neutron fluids. Extensive studies of this mode in the past two decades have investigated the dependence of its strength on the nuclear deformation and the relationship with the quadrupole collective mode and with the superfluid moment of inertia of the nucleus [3].

Recently it has been possible to study the scissors mode in Bose-Einstein condensates (BEC) of dilute gases [1]. This transverse mode of excitation has been used to demonstrate that the condensate can only flow in an irrotational manner [5,6]. This work, together with the observation of a critical velocity [7] and the occurrence of quantized vortices [8.9. has provided conclusive evidence for the superfluidity of a trapped condensate. The scissors mode of a trapped BEC has been formally linked to the quadrupole operator and to the moment of inertia of the superfluid system [10].

In condensed gases the 'scissors' motion is an irrotational oscillation of the condensate relative to the static trapping potential. The deformation of the system can be controlled independently using the trap frequencies (unlike nuclei). The most striking difference between the scissors mode in nuclei and in condensed gases is that the nuclear system is effectively at $T=0$ and the scissors excitation is fragmented because of the interference of the orbital with the spin motion, whereas in a trapped BEC it is possible to control the temperature of the gas, and hence change the fraction $N_{0} / N$ of condensed atoms.

The spectroscopy of the low energy collective modes of the condensate has been extended to include measurements of damping rates and frequency shifts as a function of temperature [11,12]. These finite temperature measurements test dynamical aspects of the BEC theory that require higher order terms in the calculations. As yet the results have not been fully explained [13].

In this paper we report a systematic study of the temperature dependent damping and frequency shifts of the scissors mode excitation in a trapped condensed gas. After a brief description of the experimental procedure, we present the scissors mode data for the frequency shifts and damping rates. We then compare our results with the available theoretical calculations for other collective modes. Finally we show how the scissors mode frequency shifts are related to quenching of the moment of inertia of the boson gas.

We excite the scissors mode using the technique described in our previous paper [6]. In summary we prepare atoms at the desired temperature $T$ in an untilted TOP trap $\left(\omega_{x}=\omega_{y}=126 \mathrm{~Hz}, \omega_{z}=\sqrt{8} \omega_{x}\right)$. We then adiabatically tilt the confining potential by a small angle $\phi=3.4^{\circ}$ about the $\mathrm{y}$ axis (this reduces $\omega_{z}$ by $\sim 2 \%$ ). We then suddenly flip the trap angle to $-\phi$ to excite the scissors mode in the xz plane with an amplitude $\theta_{0}=2 \phi$, about the new equilibrium position. The condensate oscillates at a single frequency $\omega_{s c}$ (Fig. 1 (a)). The low temperature limit of the condensate frequency is the hydrodynamic value $\omega_{s c}=\sqrt{\omega_{z}^{2}+\omega_{x}^{2}}$ 河. The thermal component oscillates at two frequencies $\omega_{+}$and $\omega_{-}$, in the collisionless limit $\omega_{+}=\omega_{z}+\omega_{x}$ and $\omega_{-}=\omega_{z}-\omega_{x}$. The high-lying frequency $\omega_{+}$corresponds to an irrotational quadrupole velocity flow $(\nabla \times \vec{v}=0)$ that is the classical counterpart of the superfluid irrotational oscillation. The low-lying frequency $\omega_{-}$of the thermal cloud is related to rotational flow, and the absence of a similar mode for the BEC is the key feature that indicates superfluidity.

To observe the scissors mode oscillation for the thermal component we imaged the atomic cloud in the trap using destructive absorption imaging. The images of the cloud in the trap were fitted with a 2D Gaussian distribution from which we extract the cloud angle as a function of time (both above and below the critical temperature). 
To observe the condensate we switch off the trapping potential at different times during the scissors mode oscillation, and allow the cloud to expand for $14 \mathrm{~ms}$ before taking an image. The thermal cloud expands isotropically while the BEC component has an elongated (prolate) shape (Fig. 11 (b)). The angle of the expanded BEC component is extracted by fitting the two component profile with a 2D double Gaussian distribution [14]. In this way we are able to separate the information on the thermal cloud scissors mode and the BEC scissors mode.

The temperature of the ultracold atomic sample is extracted from a 2D Gaussian fit to the wings of the expanded thermal component using the procedure well described in 15. For temperatures below $0.5 T_{0}$ when the thermal cloud is not visible, we deduced the temperature from the final rf by extrapolating the best fit curve for the available temperature vs rf cut frequency data. To determine the scaled temperature $T / T_{0}$ we need to calculate the critical temperature $T_{0}(N)$ at each final rf cut - in evaporation each rf cut gives a different final number of atoms $N$ and hence different $T_{0}(N)$. We calculated $T_{0}(N)$ from the measured final number of atoms in each time-of-flight picture and the known trap frequencies, using the formula for a trapped boson gas 16 . The statistical uncertainty in $T / T_{0}$ is in the range $3-6 \%$, but increased to $10 \%$ for $T / T_{0}<0.5$ where $T$ was extrapolated. To check our temperature measurements we compared the condensate fraction vs temperature with theoretical predictions for both the ideal gas and interacting gas 117] (Fig. 11 (c)).

The measurements of the frequency and damping of the quadrupole modes in previous work [11, 12] relied on measurement of the radii of the condensate and the thermal cloud. These measurements are sensitive to the fitting model and to shot to shot noise because of their atom number dependence. However the scissors mode has very well defined initial conditions for the excitation of both the BEC and the thermal cloud and the angle oscillations of the two components can be measured independently. The angles of both condensed and thermal clouds do not depend on atom number and could be fitted very reliably, even close to $T_{0}$ to give accurate measurements of frequencies and damping.

Figure 2 shows three different scissors mode oscillations at temperatures of (a) $T / T_{0}=1.29$, (b) $T / T_{0}=0.74$ and (c) $T / T_{0}=0.53$. The measured frequencies of the thermal cloud do not change with temperature and agree very well with the collisionless frequencies, both above and below the critical temperature (see also Fig. 3). Both frequency components have the same amplitude implying that energy is shared equally between rotational and irrotational velocity flow. These thermal cloud excitations have very small damping that is independent of temperature, confirming the collisionless regime of the thermal component in our experiment (see also Fig. 4).
The scissors mode oscillation of the BEC component occurs at a single frequency $\omega_{s c}$ (Fig. 2 (c) and (b)). However, the frequency of oscillation and its damping change dramatically with temperature. The low temperature limit of the frequency was $\sim 1 \%$ higher than the hydrodynamic prediction as shown in Fig. 3. This agrees with the frequency we calculated for finite number condensate $\left(2 \times 10^{4}\right)$ using the method detailed in [18].

Figure 14 shows the experimental data for the temperature dependent damping rates. Since a finite $T$ theory of the scissors mode in a TOP trap is not yet available we have compared our experimental results with recent theories for other observed collective modes of a BEC.

Fedichev et al. 19 have calculated the Landau damping rate for the $|m=2|$ and low-lying $m=0$ modes of BEC in the usual TOP trap geometry $\left(\omega_{x}=\omega_{y}, \omega_{z}=\sqrt{8} \omega_{x}\right)$ for the experimental conditions of the JILA trap [11], that are close to those of our experiment. In the region $0.5<T / T_{0}<0.9$ their theoretical calculations give a damping rate $\Gamma_{\nu}$ for the collective mode $\nu$ that is proportional to $T / \mu$ and to the frequency of the excitation $\omega_{\nu}$ :

$$
\Gamma_{\nu}=A_{\nu} \omega_{\nu} \frac{T}{\mu}\left(n_{0} a^{3}\right)^{1 / 2}
$$

where $n_{0}$ is the peak density, $\mu$ is the chemical potential and $A_{\nu}$ is a numerical coefficient dependent on the shape of the collective excitation. For the quadrupole modes they calculated $A_{|m=2|} \approx 7$ for the $|m=2|$ and $A_{m=0} \approx 5$ for the low-lying $\mathrm{m}=0$. The numerical coefficient $A_{\nu}$ is likely to be similar for the scissors mode. Assuming that $A_{s c} \approx A_{|m=2|} \approx 7$ and scaling the results by Fedichev et al. 19] by the frequency factor $\omega_{s c} / \omega_{|m=2|}$, we obtain the theoretical damping rate for the scissors mode (Fig. 4, solid line), which is in fair agreement with our data. This numerical coefficient for the scissors mode could in principle be evaluated by the method described by Fedichev et al. [19]. They use an overlap of the wavefunction of the mode with the wavefunctions of the thermal excitations following classical trajectories within the range $\mu<T<0.9 T_{0}$. This method gives a way of calculating the damping process for a trapped BEC, the evaluation of the trajectories and the stochastic averaging is a numerical problem of moderate size. Our measurements of the damping extend to temperatures where $T \leq \mu$. In this region $0<T / T_{0}<0.5$ Fedichev et al. predict damping proportional to $(T / \mu)^{3 / 2}$ 19 but our experimental data have a weak dependence on the temperature.

In a recent paper Rusch et al. 21] have used a full second order theory to calculate the damping and frequency shift of low energy collective modes for a trapped BEC in a spherical geometry [22]. A spherical cloud has no scissors mode but it is possible to identify this mode with the quadrupole mode $l=2, m= \pm 1$. Thus we have used their calculations for the $l=2$ mode damping rate for comparison scaled by the frequency factor $\omega_{s c} / \omega_{l=2}$ 
(Fig. 田, dotted line). These numerical calculations give a weakly linear dependence of damping on temperature but the experimental data seem to follow a higher power law.

We have not included the observed decrease in damping at higher temperatures (above $0.85 T_{0}$ ) in the above discussion because the uncertainties are large for these data points. In addition the frequency shift of the scissors mode at these temperatures may lead to resonant coupling with other modes or with the thermal cloud. For example in [20] the lowering of the damping of the quadrupole modes close to the transition point, has been interpreted as a dynamical coupling between the condensed and thermal component.

The moment of inertia of a system is defined as linear response to a rotational field. Superfluids have a moment of inertia $\Theta$ less than that of a classical rigid body $\Theta_{\text {rig }}$ with the same density distribution. The scissors mode is ideally suited to studying this quenching of the moment of inertia below $T_{0}$. Zambelli and Stringari [10] linked the reduced moment of inertia of the boson gas $\Theta^{\prime}=\Theta / \Theta_{\text {rig }}$ to the quadrupole moment Fourier signal $Q(\omega)$ for the condensate at $T=0$ and the thermal cloud at $T>T_{0}$. Applying their derivation to the scissors mode gives the moment of inertia of the condensate and thermal clouds as:

$$
\Theta_{\text {cond }}^{\prime}=\frac{\left(\omega_{z}^{2}-\omega_{x}^{2}\right)^{2}}{\omega_{s c}^{4}} ; \Theta_{\text {therm }}^{\prime}=\frac{\left(\omega_{z}^{2}-\omega_{x}^{2}\right)^{2}}{\omega_{+}^{2} \omega_{-}^{2}}
$$

We can use Eq. 2 to deduce the moment of inertia for the condensate and thermal cloud separately. The moment of inertia for the thermal component is consistent with the rigid body value $\Theta_{\text {therm }}^{\prime}=1$ for all measured $T$. For the point at $0.99 T_{0}$ in Fig. 3 , the condensate scissors mode has a frequency such that its moment of inertia is close to that of a rigid body. Whereas as $T \rightarrow 0$ the condensate has a quenched irrotational value of the moment of inertia characteristic of a superfluid. The lower temperature points are in good agreement with the hydrodynamic frequency and the reduced moment of inertia assumes the superfluid value for our geometry $\Theta_{\text {cond }}^{\prime}=\epsilon^{2}=0.6$, where $\epsilon=\left(\omega_{z}^{2}-\omega_{x}^{2}\right) /\left(\omega_{z}^{2}+\omega_{x}^{2}\right)$ is the anisotropy of the trap [10].

In conclusion we have made precise measurements of the frequency and damping of the scissors mode as a function of temperature. The observed damping for low temperatures is consistent with that expected from models of Landau damping, where the damping rate is proportional to the mode frequency. At temperatures just below $T_{0}$ the experimental damping rate was less than predicted. The frequency shift as a function of temperature is consistent with a quenching of the moment of inertia as the temperature is reduced because of the superfluidity of the gas. We await a theoretical calculation of this shift for comparison.
We would like to thank S. Stringari and F. Zambelli, N. Lo Iudice, and all the members of the Oxford theoretical BEC group in particular K. Burnett, M. Davis, M. Rusch, S. Morgan, for many very useful discussions. We are also grateful to A. Minguzzi for providing the theoretical prediction of Fig. 11 (c).

This work was supported by the EPSRC and the TMR 'Cold Quantum Gases' network program (No. HPRNCT-2000-00125). O.M. Maragò acknowledges the support of a Marie Curie Fellowship, TMR program (No. ERB FMBI-CT98-3077).

[1] D. Bohle, A. Richter, W. Steffen, A.E.L. Dieperink, N. Lo Iudice, F. Palumbo, and O. Scholten, Phys. Lett. 137B, 27 (1984).

[2] N. Lo Iudice and F. Palumbo, Phys. Rev. Lett. 41, 1532 (1978); E. Lipparini and S. Stringari, Phys. Lett. 130B,139 (1983).

[3] N. Lo Iudice, Phys. Part. Nucl., 28, 556(1997) and Rivista del Nuovo Cimento, 23, 1(2000).

[4] M.H. Anderson et al., Science 269, 198 (1995); K.B. Davis et al., Phys. Rev. Lett. 75, 3969 (1995).

[5] D. Guéry-Odelin and S. Stringari, Phys. Rev. Lett. 83, 4452 (1999).

[6] O.M. Maragò, et al., Phys. Rev. Lett. 84, 2056 (2000).

[7] C. Raman et al. Phys. Rev. Lett. 83, 2502 (1999); A.P. Chikkatur, et al. Phys. Rev. Lett. 85, 483 (2000).

[8] M.R. Matthews et al. Phys. Rev. Lett. 83, 2498 (1999).

[9] K.W. Madison et al. Phys. Rev. Lett. 84, 806 (2000).

[10] F. Zambelli and S. Stringari, Phys. Rev. A 63, 033602 (2001); S. Stringari, Phys. Rev. Lett. 76, 1405 (1996).

[11] D.S. Jin, M.R. Matthews,J.R. Ensher, C.E. Wieman and E. A. Cornell, Phys. Rev. Lett. 78, 764 (1997).

[12] D.M. Stamper-Kurn, H.-J. Miesner, S. Inouye, M. R. Andrews, and W. Ketterle, Phys. Rev. Lett. 81, 500 (1998).

[13] D.A.W. Hutchinson, R.J. Dodd, and K. Burnett, Phys. Rev. Lett. 81, 2198 (1998).

[14] We have also used a 2D fitting distribution made of a Gaussian profile for the thermal component and a Thomas-Fermi profile for the condensate. This gave the same result for the scissors angle, confirming that this is not affected by the choice of the model to describe the cloud profile. The condensate fraction was instead systematically lower of about $10 \%$ compared to the Gaussian profile fitting.

[15] J.R. Ensher et al., Phys. Rev. Lett. 77, 4984 (1996); W. Ketterle in Bose-Einstein Condensation in Atomic Gases, Proceedings of the International School of Physics "Enrico Fermi", edited by M. Inguscio, S. Stringari and C.E. Wieman, (IOS Press, Amsterdam, 1999).

[16] V. Bagnato, D.E. Pritchard, and D. Kleppner, Phys. Rev. A 35, 4354 (1987).

[17] A. Minguzzi et al., J. Phys. (Condens. Matter) 9, (1997) L33-L38. 
[18] M.O. da C. Pires and E.J.V. de Passos, J.Phys.B. 33 3929 (2000).

[19] P.O. Fedichev, G.V. Shlyapnikov, and J.T.M. Walraven, Phys. Rev. Lett. 80, 2269 (1998).

[20] B. Jackson and C.S. Adams, cond-mat/0009468.

[21] M. Rusch, S.A. Morgan, D.A. Hutchinson, and K. Burnett, Phys. Rev. Lett. 85, 4844 (2000).

[22] Both the calculation of the frequency and damping rate as a function of $T$ requires detailed knowledge of the frequencies of all the significant fundamental modes of the condensate which mix with the mode under study. Such calculations are being carried out for the scissors mode by extending the finite $T$ theory of the BEC developed by Rusch et al. 21] to the case of the TOP trap (rather than a spherically symmetric potential).
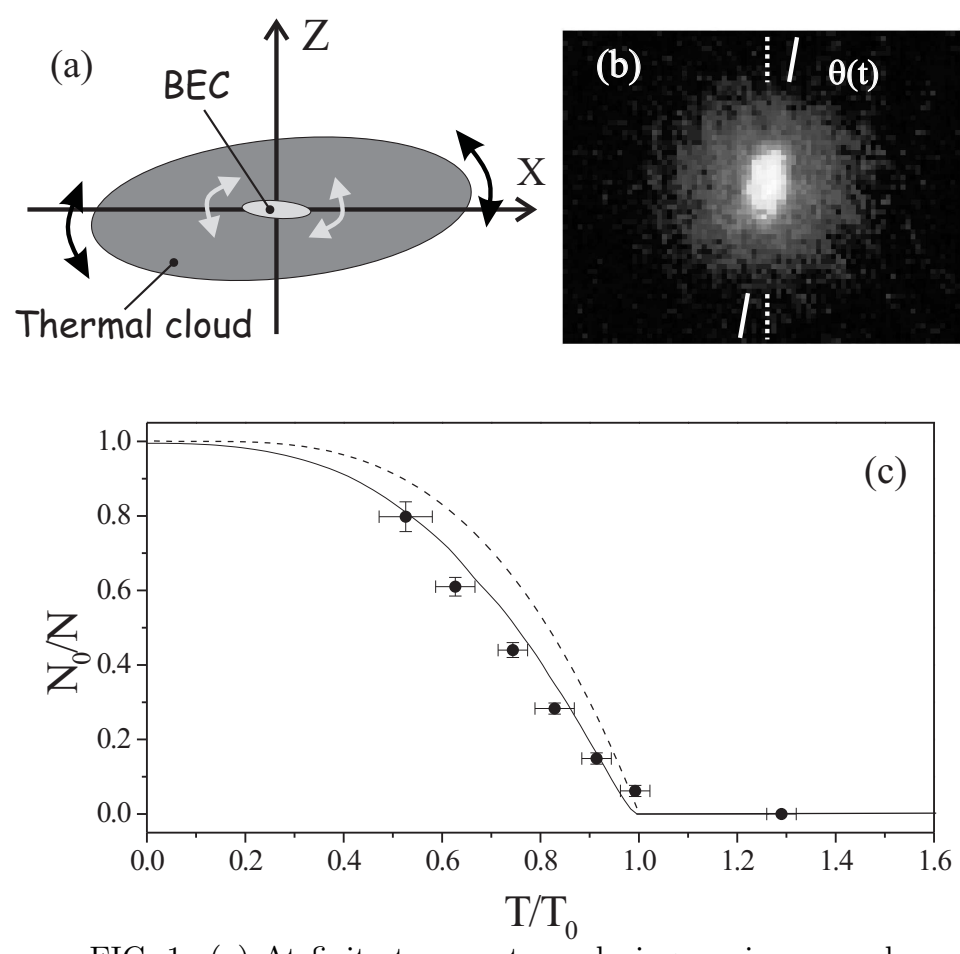

FIG. 1. (a) At finite temperature, during a scissors mode oscillation, the condensate and the thermal cloud oscillate at their characteristic frequencies. (b) Typical time of flight (TOF) picture during a scissors mode oscillation at finite temperature: the thermal cloud is isotropically expanded, while the condensate has an elongated shape. From a 2D double Gaussian distribution fit to the picture we extract the angle of the BEC. For this picture $N_{0} / N=0.14, T / T_{0}=0.91$ and $\theta=-8.5^{\circ}$ with respect to the vertical axis. (c) Condensate fraction as a function of the reduced temperature $T / T_{0}$. The solid line is the interacting theory prediction made by A. Minguzzi et al. for our experimental conditions. The dashed line is the ideal trapped gas cubic law prediction. The experimental points (solid circles) are obtained from TOF images as explained in the text.

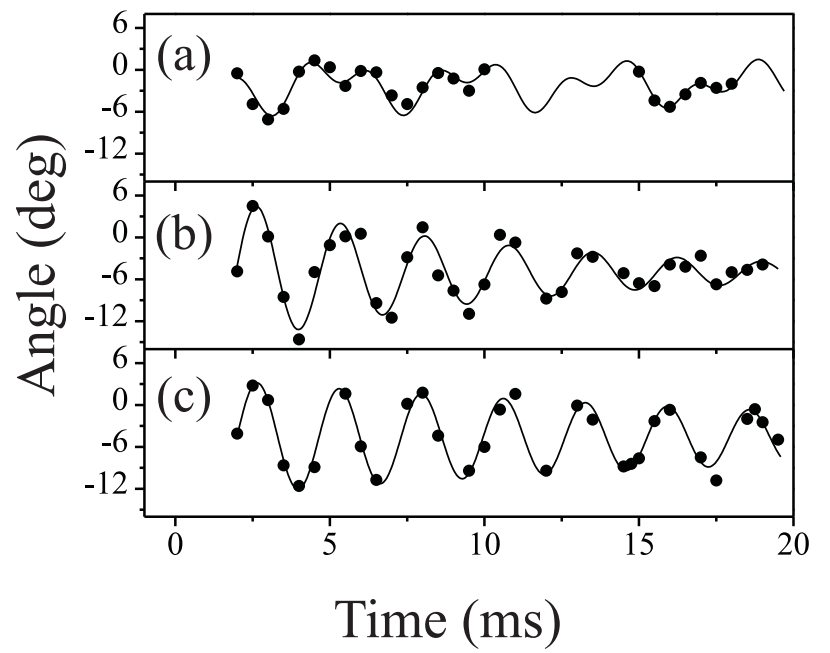

FIG. 2. Angle versus time during the scissors mode. (a) Thermal cloud, $T / T_{0}=1.29$. The scissors mode is characterized by two frequencies of oscillation with equal amplitude. (b) Condensate, $T / T_{0}=0.74$. A heavily damped BEC oscillation is observed at a single frequency, below that of hydrodynamic theory. (c) Condensate, $T / T_{0}=0.53$. Lightly damped BEC oscillation at a frequency that agrees well with the hydrodynamic value. 


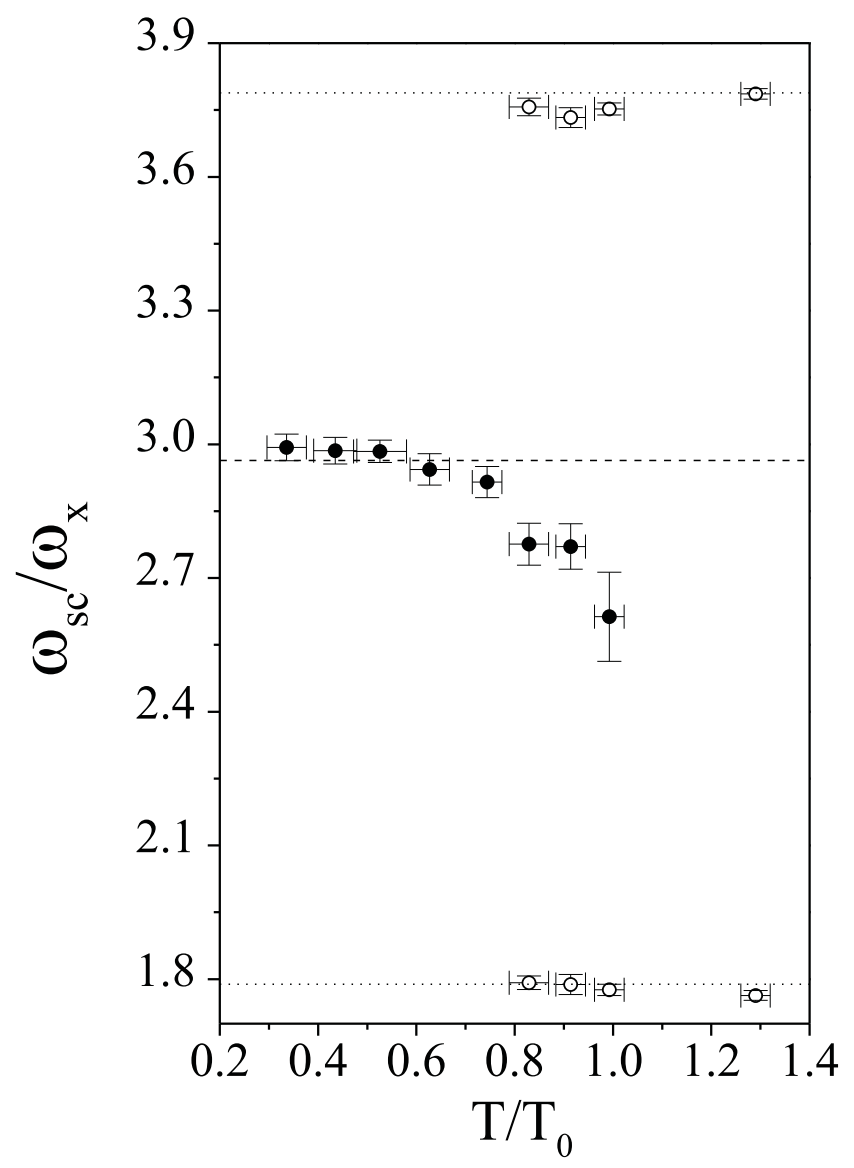

FIG. 3. Temperature dependent frequency shift of the scissors excitation of the condensate (solid circles) and thermal component (open circles). Significant negative shifts from the hydrodynamic prediction (dashed line) of the condensate scissors mode frequency are observed as the temperature increases. The low temperature frequencies are systematically higher than the hydrodynamic value by $\sim 1 \%$ due to the finite number of atoms. The two frequencies of the scissors mode of the thermal component do not appear to be temperature dependent and are in good agreement with collisionless predictions (dotted lines). The frequency spectrum has been normalized by the radial trap frequency.

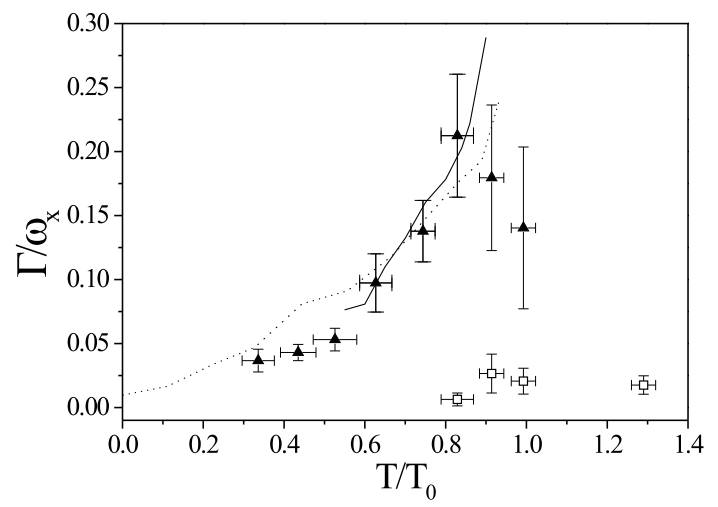

FIG. 4. The temperature dependence of the damping rate $\Gamma$ normalized to the radial trap frequency $\omega_{x}$ for the condensate (solid triangles) and thermal component (open squares). The solid line is the theoretical damping rate of the $|m=2|$ mode calculated by Fedichev et al. (which agrees well with the experimental data obtained at JILA for that mode) multiplied by the frequency scaling factor $\omega_{s c} / \omega_{|m=2|}$ for the scissors mode. The dotted line is the prediction for the $l=2$ mode by Rusch et al. in a spherical geometry rescaled by the frequency factor for the scissors mode. 